\title{
DISERTACIONES
}

ENSAYOS

Anuario electrónico de estudios en Comunicación Social

ISSN: $1856-9536$

Doi: dx.doi.org/10.12804/disertaciones.09.02.2016.02

Volumen 9, Número 2 / julio-diciembre 2016

Versión PDF para imprimir desde

http://revistas.urosario.edu.co/index.php/disertaciones

Para citaresteartículo:Saperas, E. (2016). Cuatro décadas deinvestigación comunicativa en España. Los procesos de institucionalización y de profesionalización de la investigación (1971-2015). Anuario Electrónico de Estudios en Comunicación Social "Disertaciones", 9(2), 27-45. Doi: dx.doi.org/10.12804/disertaciones.09.02.2016.02

\section{CUATRO DÉCADAS DE INVESTIGACIÓN COMUNICATIVA EN ESPAÑA. LOS PROCESOS DE INSTITUCIONALIZACIÓN Y DE PROFESIONALIZACIÓN DE LA INVESTIGACIÓN (1971-2015)*}

Four Decades of Communication Research in Spain. The Processes of Institutionalization and Professionalization of Research (1971-2015)

Quatro décadas de investigação comunicativa na Espanha. Os processos de institucionalização e de profissionalização da investigação (1971-2015)

Saperas Lapiedra, Enric. Universidad Rey Juan Carlos (España) enric.saperas@urjc.es

Fecha de recibido: 22 de febrero de 2016

Fecha de aceptado: 29 de febrero de 2016

\footnotetext{
Este ensayo se inscribe en el marco del proyecto de investigación 25 años de investigación sobre comunicación en España. Producción científica, comunidad académica y contexto institucional (Incomes-25) del Plan Estatal de Investigación Científica y Técnica de Excelencia (2013-2016) (CSO2013-40684-P). IP: Manuel Martínez-Nicolás.
} 


\title{
DISERTACIONES
}

ENSAYOS

Anuario electrónico de estudios en Comunicación Social

ISSN: $1856-9536$

Doi: dx.doi.org/10.12804/disertaciones.09.02.2016.02

Volumen 9, Número 2 / julio-diciembre 2016

Versión PDF para imprimir desde

\section{RESUMEN}

Una disciplina científica se define por su contexto institucional y por la organización intelectual del campo de investigación. La finalidad de este ensayo es describir el primer elemento que delimita la formación de un campo disciplinar: el contexto institucional. En este caso, se observarán los procesos de formación de la comunidad científica, de institucionalización y de profesionalización que han configurado la investigación comunicativa española desde 1971 hasta la actualidad. Esta investigación ha sido objeto de estudio en numerosas ocasiones y en periodos muy diversos pero mayoritariamente se ha estudiado su producción científica, los marcos teóricos y metodológicos de referencia y los temas de investigación de mayor presencia. Sin embargo, los aspectos institucionales y de profesionalización conforman los factores que diseñan el modelo académico y de investigación desarrollado en cada sistema universitario estatal. La investigación comunicativa está plenamente consolidada en España, ha desarrollado un modelo que presenta similitudes pero también rasgos diferenciales respecto a los países del entorno. Sin conocer estos rasgos propios será difícil poder dar respuesta al extraordinario reto que se ha planteado en los últimos años y que se debe resolver de forma ineludible: la internacionalización.

Palabras clave: investigación comunicativa, institucionalización, profesionalización, comunidad académica, disciplina comunicativa.

\begin{abstract}
A scientific discipline is defined by its institutional context and intellectual organization of field research. The purpose of this paper is to describe the first element that defines the formation of a disciplinary field in Social Scienes: the institutional context. In this case the formation process of the scientific community, institutionalization and professionalization that have shaped the Spanish communication research from 1971 to the present day will be observed. The Spanish communication research has been studied on numerous occasions and in very different periods but mostly has been studied his scientific, theoretical and methodological frameworks and research topics of greater presence. However, are the institutional and professional aspects that make up the elements that design an academic and research model developed in each university system. Communication research is fully consolidated in Spain, it has developed a model that has similarities but also distinctive features compared to neighboring countries. Without knowing these traits will be difficult to respond to the extraordinary challenge raised in recent years and that must be resolved so inescapable: internationalization.
\end{abstract}

Keywords: Communication Research, Institutionalization Process, Research Professionals, Academic Community, Communicative Discipline.

\section{RESUMO}

Uma disciplina científica se define pelo seu contexto institucional e pela organização intelectual do campo de pesquisa. A finalidade deste ensaio é descrever o primeiro elemento que delimita a formação de um campo disciplinar: 


\section{DISERTACIONES}

ENSAYOS

Anuario electrónico de estudios en Comunicación Social

ISSN: $1856-9536$

Doi: dx.doi.org/10.12804/disertaciones.09.02.2016.02

Volumen 9, Número 2 / julio-diciembre 2016

Versión PDF para imprimir desde

http://revistas.urosario.edu.co/index.php/disertaciones

o contexto institucional. Neste caso se observarão os processos de formação da comunidade científica, de institucionalização e profissionalização que têm configurado a investigação comunicativa espanhola desde 1971 até a atualidade. Esta investigação tem sido objeto em numerosas ocasiões e em períodos muito diversos, mas maioritariamente se tem estudado a sua produção científica, os marcos teóricos e metodológicos de referência e os temas de investigação de maior presença.

No entanto, os aspectos institucionais e de profissionalização conformam os fatores que desenham o modelo acadêmico e de investigação desenvolvido em cada sistema universitário estatal. A investigação comunicativa está plenamente consolidada na Espanha, tem desenvolvido um modelo que apresenta similitudes, mas também rasgos diferenciais respeito aos países do entorno. Sem conhecer estes rasgos próprios será difícil poder dar resposta ao extraordinário reto apresentado nos últimos anos e que devesse resolver de forma inevitável: a internacionalização.

Palavras-chave: Investigação comunicativa, institucionalização, profissionalização, comunidade acadêmica, disciplina comunicativa.

\section{Introducción}

Recientemente, Craig Calhoun (2011) hacía referencia a la necesidad de una nueva responsabilidad social de la investigación sobre comunicación mediática al decir que parece confirmarse que "los temas estudiados por la investigación comunicativa no solo son importantes en sí mismos sino que lo son porque están siendo socialmente reconocidos como importantes" (p. 149). La influencia de los medios de comunicación, la innovación tecnológica y los cambios en la estructura de los sistemas mediáticos se han convertido en un tema de interés público pocas veces igualado en el pasado. Quizá solo en los años posteriores a la Gran Guerra, con el advenimiento de la industria del audiovisual, la internacionalización de los mercados cinematográficos y de música popular y la propaganda política los medios de comunicación constituyeron un tema de atención pública y de interés social similar al actual. En el actual contexto, investigar cómo se organiza la investigación comunicativa, cómo ha evolucionado en la últimas décadas y cuál ha sido la formación del campo disciplinar no constituye un simple ejercicio de nostalgia académica sino que brinda la oportunidad de conocer sistemáticamente un ámbito central en la producción científica y la transferencia de conocimiento en las ciencias sociales en su estadio actual.

En este ensayo, se propone llevar a cabo este objetivo en el caso español. Y se hará mediante la observación de los procesos de institucionalización y de profesionalización de la investigación comunicativa desde sus inicios en las facultades de Ciencias de la Información creadas en los últimos años de la dictadura hasta la actualidad. 


\section{DISERTACIONES}

Anuario electrónico de estudios en Comunicación Social

ISSN: 1856-9536

Doi: dx.doi.org/10.12804/disertaciones.09.02.2016.02

Volumen 9, Número 2 / julio-diciembre 2016

Versión PDF para imprimir desde

http://revistas.urosario.edu.co/index.php/disertaciones

\section{La investigación comunicativa en España como objeto de estudio}

El estudio y la evaluación de la investigación comunicativa en España ha sido un tema de interés constante por parte de numerosos investigadores que han generado, en su conjunto, una notable literatura académica. Este es un aspecto que diferencia a España del resto de países del entorno en los que este tipo de literatura no es muy abundante. En definitiva, en 2016 no se puede prescindir del conjunto de artículos, libros, textos de trabajo y de comunicaciones en congresos que desde la publicación en 1981 de Teorías de la Comunicación de Miquel de Moragas hasta la actualidad han descrito y diagnosticado esta modalidad de la investigación, su estatus académico y su definición como campo disciplinar. Sin excesivo esfuerzo, es fácil enumerar algunos de los aportes más significativos y de referencia obligada. La lectura del libro anteriormente citado de Miquel de Moragas, cuya publicación coincidió con el décimo aniversario de la creación facultades de Ciencias de la Información, constituye un referente indiscutido para la difusión y organización del campo disciplinar en España, aporta una visión sistemática de los precedentes históricos y de su cronología, así como sus procesos de institucionalización universitaria y académica. Los estudios y artículos sobre este ámbito de Daniel Jones constituyen un referente imprescindible (Jones, 1997, 1998). De forma similar, y en el ámbito de la entonces llamada "escuela catalana" creada en la Universidad Autónoma de Barcelona, dos monografías de Josep Gifreu y de Jordi Berrio sobre la investigación en Cataluña (Gifreu, 1988; Berrio, 1997) ocupan un lugar de referencia para conocer los precedentes de los estudios de comunicación a lo largo de siglo xx, la devastación universitaria de la dictadura y la formación de la investigación en tiempos de la restauración democrática. Con la entrada en el siglo xx y ya en periodo de consolidación de la investigación comunicativa, son de lectura imprescindible los dos artículos de Manuel Martínez-Nicolás (2006; 2009) que hasta la actualidad pueden considerarse como la interpretación más compleja y exhaustiva de cómo la investigación comunicativa española ha alcanzado en la actualidad una situación de maduración (en palabras del propio investigador). Y por último, resulta también de lectura obligada el texto de Roberto de Miguel (2007) en el que se ofrece una descripción sistemática sobre la sociología de la comunicación en España. Por otra parte, los estudios académicos sobre la investigación comunicativa también han explorado con éxito campos disciplinares particulares que son de consulta obligada si se quiere conocer la investigación española sobre periodismo y cultura periodística (Martínez-Nicolás, 2006; Humanes, 2007), la economía política de la comunicación (Almirón \& Reig, 2011), el ciberperiodismo (Salaverría, 2007) o el proceso de debate y formación de las teorías de la comunicación en España (García-Jiménez, 2007, 2015; Rodrigo \& García-Jiménez, 2010; Piñuel, 2011; Silva \& San-Eugenio, 2014). También destacan los estudios recientes, en su mayoría a partir de 2011, de la producción científica publicada en las revistas especializadas españolas sobre comunicación mediática y sus índices de impacto (Martínez-Nicolás \& Saperas, 2011; Masip, 2011; Fernández-Quijada \& Masip, 2013).

Este interés por "investigar la investigación" ha dado lugar en los últimos años a la creación de grupos de investigación (o líneas estables encuadradas en grupos de investigación) especializados en este ámbito y que cuentan con proyectos competitivos sobre este objeto de estudio. En 2009 el Grupo de Estudios Avanzados de la Comunicación GEAC decidió iniciar esta línea de investigación centrada en la historia y análisis de la investigación comunicativa y en el estudio del contenido de las revistas fuente en el caso español y de revistas de referencia internacional (Martínez-Nicolás \& Saperas, 2011; Saperas \& Carrasco-Campos, 2015). Más recientemente, también 


\section{DISERTACIONES}

ENSAYOS

Anuario electrónico de estudios en Comunicación Social

ISSN: $1856-9536$

Doi: dx.doi.org/10.12804/disertaciones.09.02.2016.02

Volumen 9, Número 2 / julio-diciembre 2016

Versión PDF para imprimir desde

http://revistas.urosario.edu.co/index.php/disertaciones

se ha incorporado a este campo de estudio el grupo sobre Mediación Dialéctica de la Comunicación Social (MDSC), en este caso más centrado en el ámbito específico de la Teoría de la Comunicación y en la formación del campo disciplinar.

Se trata, pues, de una línea de investigación consolidada y con un buen potencial de desarrollo futuro que ha dado lugar en enero 2015 a la formación del Grupo de Trabajo en Historia de la investigación sobre comunicación de la Asociación Española de Investigación de la Comunicación (AE-IC).

En estos documentos académicos, y en otros muchos de interés de los que se harán referencia más adelante, el lector podrá conocer de forma exhaustiva los precedentes, las primeras tradiciones de investigación y el estado actual de este sector de la investigación comunicativa española. Por ello, en este ensayo se remitirá a estos textos de referencia para su conocimiento exhaustivo y sistemático.

\section{Los inicios de la investigación en España: algunos elementos diferenciadores respecto al entorno europeo más cercano}

La investigación comunicativa en España se inició con la formación de un modelo institucional y disciplinar, en muchos aspectos, similar al resto de países del sur y centro de Europa pero, en otros muchos, claramente diferenciado. A lo largo de las cuatro décadas transcurridas se ha pugnado en la comunidad académica del campo entre un sector arraigado en el modelo de origen y comprometido con la evolución interna del modelo fundacional y un sector que ha querido situar la investigación española en lo que se podrían denominar como estándares internacionales. Por el contrario, en la docencia y formación profesional son similares a las del entorno de la Europa mediterránea. En este ámbito de formación de profesionales de la comunicación, y sobre todo en lo que concierne a la formación de periodistas, las diferencias son escasas salvo en cuestiones relativas a la denominación de los títulos universitarios de las áreas de conocimiento, y en cuanto a la naturaleza de los centros académicos que en cada país se corresponden con su estructura universitaria, profesional y sindical.

A continuación se verán algunos rasgos diferenciales surgidos en unos orígenes todavía presentes. En el país, se trata de una investigación tardía debido a la dictadura y al aislacionismo internacional y que, a diferencia de la mayoría de países del sur y del centro de Europa, no disponía de precedentes significativos ni de orden institucional (estudios universitarios, centros de investigación, revistas científicas, escuelas de periodismo, escuelas de cine) ni de carácter científico más allá de una significativa labor ensayística de algunos pensadores y profesionales de referencia que reflexionaron sobre el advenimiento de la cultura de masas, el protagonismo de los media en la sociedad industrial, el periodismo o la cinematografía. El libro Teorías de la Comunicación de Miquel de Moragas continúa siendo el estudio de referencia para el conocer este periodo previo al inicio de la investigación en España (Moragas, 1981). Como consecuencia del contexto histórico español, y a diferencia de Francia, Italia y Alemania (Saperas, 1997), los tres modelos más influyentes en el periodo inicial de la investigación española, y esto es muy significativo, no se habría producido recepción alguna del empirismo positivista, y especialmente, de la investigación del estructural funcionalismo. En las fases iniciales de la investigación, y de forma contraria a Francia, Italia, Alemania y también Portugal, el empirismo carecerá de influencia tanto en la formación profesional como en la investigación en los primeros centros universitarios dedicados a los estudios mediáticos. Serán las humanidades 


\section{DISERTACIONES}

Anuario electrónico de estudios en Comunicación Social

ISSN: 1856-9536

Doi: dx.doi.org/10.12804/disertaciones.09.02.2016.02

Volumen 9, Número 2 / julio-diciembre 2016

Versión PDF para imprimir desde

http://revistas.urosario.edu.co/index.php/disertaciones

(filosofía, historia, derecho, arte, filología, lingüística) la matriz de la investigación por dos motivos. El primero, la mayor parte del nuevo profesorado que impartirá periodismo, imagen o publicidad en las nuevas facultades de Ciencias de la Información serán reclutados en este ámbito. El segundo, las ciencias sociales, y de forma preferente la sociología, la ciencia política y, en menor medida, la psicología social, también sufrieron la represión académica por parte de la dictadura. De forma similar a los estudios de comunicación, la sociología y la ciencia política desarrollarán los actuales centros universitarios propios en el periodo 1975-1989. En este sentido, se quiere destacar la relevancia del contexto general de la universidad española de las décadas de los años setenta y ochenta para comprender el porqué, y el nuevo marco institucional en la creación de las facultades de Comunicación y de sus licenciaturas específicas. Estas solo se explican si se tiene en consideración que en esas dos décadas se procederá a reestructurar institucionalmente las facultades y titulaciones universitarias en el conjunto de las ciencias sociales. En esta reestructuración, y al ser un tipo de disciplina científica inexistente en España en aquel momento, la creación de las facultades de Comunicación debe ser interpretada como un primer paso hacia la reforma institucional en la formación universitaria de las ciencias sociales. A continuación, se mencionarán dos ejemplos de este contexto de transformación en las ciencias sociales externos a las ciencias de la comunicación. En la Universidad Complutense de Madrid, también en 1971, la Facultad de Ciencias Políticas y Económicas, creada en 1943, se escindía en dos centros: Ciencias Políticas y Sociología, y Ciencias Económicas y Empresariales, mientras que en 1973 se reestructura la Facultad de Filosofía y Letras, en la que Psicología quedará integrada en la Facultad de Filosofía y Ciencias de la Educación hasta febrero de 1980, en que se constituye como facultad específica. En un segundo caso, en la Universidad Autónoma de Barcelona la reestructuración se producirá en los ochenta con la creación de la Facultad de Ciencias Políticas y de Sociología, en 1985, y de la Facultad de Psicología en 1989.

El inicio de la investigación se producirá, en palabras de Moragas (1981), en unas "condiciones peculiares" que parecían generar el convencimiento de que la bibliografía internacional "no podía aplicarse sino después de numerosos retoques, a la interpretación de nuestra situación comunicativa y cultural” (p. 217). Esta posición de partida será de gran relevancia para el desarrollo de la primera investigación, y evidente para sus desarrollos posteriores hasta estos días. Probablemente, en esta posición de inicio, una deficiencia en la formación de las ciencias sociales ha determinado ciertas carencias en la producción científica sobre comunicación mediática y una marcada falta de trascendencia internacional de esta producción (Martínez-Nicolás, 2006). En pocas palabras, la investigación comunicativa española partió de un marco institucional más cercano a una autosuficiencia comunicológica (Martínez-Nicolás, 2006) que, a la matriz de las ciencias sociales, particularmente de la sociología, y como recordaba Moragas (1981), "hablar de la investigación de las comunicaciones de masas es, en la mayor parte de los casos y de los países, hablar de investigación sociológica” (p. 22). Este punto de partida será decisivo para la institucionalización de la investigación comunicativa al recluirse mayoritariamente en las recién creadas facultades de Ciencias de la Información.

Y este será un rasgo diferencial de gran relevancia en el caso español, si se compara con los sistemas universitarios francés, italiano o portugués. En esos países, y mediante modelos propios de cada sistema universitario, la institucionalización de la formación profesional y de la investigación se ha caracterizado por la diversidad de centros universitarios y la coordinación entre facultades, escuelas de enseñanza superior, institutos universitarios y departamentos de ciencias de la comunicación, ciencias sociales, humanidades, y filosofía y letras. En definitiva, en los países del entorno más cercano, la investigación se ha formalizado mediante sistemas mixtos en los 


\section{DISERTACIONES}

ENSAYOS

Anuario electrónico de estudios en Comunicación Social

ISSN: 1856-9536

Doi: dx.doi.org/10.12804/disertaciones.09.02.2016.02

Volumen 9, Número 2 / julio-diciembre 2016

Versión PDF para imprimir desde

http://revistas.urosario.edu.co/index.php/disertaciones

que participan tanto centros específicos de ciencias de la comunicación como centros de ciencias sociales y de filosofía y letras o humanidades. En la formación profesional, este sistema híbrido suele dar paso a titulaciones específicas de primer y segundo ciclos (grado) o tan solo de segundo ciclo (posgrado). De esta manera, se mencionan algunos rasgos de los sistemas universitarios del entorno en lo concerniente a la formación de investigadores y a la propia práctica académica de la investigación. En el caso francés, las diferencias institucionales se sitúan en los ciclos formativos con un predominio de escuelas superiores (segundo ciclo) y en menor medida de institutos universitarios (primer ciclo) que conviven con facultades genéricas de ciencias de la información y de la comunicación. En el caso de las escuelas superiores, y de forma especialmente significativa en las dedicadas a la formación de periodistas, se instituye una coordinación de los planes de estudio y de los criterios de admisión con las asociaciones profesionales y sindicales. En cuanto a la investigación, cuyo ciclo formativo se realiza mediante un Master Recherche de dos años y el doctorado de tres años, se organizan de forma compartida tanto por facultades y escuelas universitarias de Ciencias de la Información y de la Comunicación y por institutos universitarios como por facultades de Ciencias Sociales y de Humanidades, así como mediante Centros Superiores de Investigación en Ciencias Sociales y Humanas. En el caso italiano, la enseñanza y la investigación en comunicación se encuentra organizada mediante instituciones universitarias de una gran diversidad. En cuanto a la formación de los profesionales de la comunicación, se realiza mediante centros universitarios profesionales, que en el caso del periodismo cuentan con sistemas de coordinación y de reconocimiento de titulaciones por parte de los sindicatos nacionales (Laurea y Master), institutos universitarios (Laurea y Master), y Facultades de Ciencias de la Comunicación con ciclos de especialización profesional en periodismo, comunicación audiovisual y publicidad; todos ellos con título específico de Ciencias de la Comunicación (Laurea). En cuanto a la investigación, se realiza mediante una formación de segundo ciclo en facultades de Ciencias de la Comunicación, y en estudios específicos en facultades de Filosofía y Letras, facultades de Sociología, Ciencias Políticas y Ciencias de la Educación. La formación para la investigación se organiza mediante un Master de ॥ Nivel (formación para la investigación de un año posterior la Laurea specialistica) y, posteriormente, un doctorado de investigación de tres años de duración. En Portugal, la formación universitaria se organiza mediante dos tipos de centros universitarios: las facultades y las escuelas politécnicas (más próximas a la estricta formación profesional). El rasgo más significativo en este modelo es el hecho de que tanto la docencia universitaria de primer ciclo (Licenciatura), de segundo ciclo (Mestre universitário) y el doctorado son responsabilidad de departamentos de Ciencias de la Comunicación (específicos o conjuntos con ciencias sociales y humanidades), que disponen de plena autonomía en la gestión de las titulaciones de comunicación y son responsables, prácticamente en exclusiva, de las actividades de investigación en comunicación. Sin embargo, se encuentran institucionalmente integrados en facultades de Ciencias Sociales, Humanidades, facultades de Filosofía y Letras o en escuelas politécnicas.

\section{Primer marco institucional (1971-1982): las facultades de Ciencias de la Información}

En el caso español, y debido a las especificidades de su contexto político y universitario, el modelo institucional de formación y de investigación se desarrolló mediante la creación de centros universitarios específicos que han 


\section{DISERTACIONES}

ENSAYOS

Anuario electrónico de estudios en Comunicación Social

ISSN: 1856-9536

Doi: dx.doi.org/10.12804/disertaciones.09.02.2016.02

Volumen 9, Número 2 / julio-diciembre 2016

Versión PDF para imprimir desde

logrado una notable eficiencia tanto en su oferta de titulaciones (de éxito social y profesional innegable) como, aunque en menor media, en su producción científica.

La investigación comunicativa en España, y de forma similar a la investigación portuguesa, se iniciará y consolidará de forma muy tardía respecto al entorno europeo, debido a la dictadura que durante cuatro décadas impondrá la represión y el control sistemático de la ciencia en España. Solo en el último periodo de decadencia de la dictadura, y a partir de la necesidad de reformar el régimen ante los inevitables cambios políticos que implicaría la muerte del dictador, se decidió apostar por una reforma de la formación profesional de los periodistas mediante su inserción en el sistema universitario y su necesaria adaptación a la modernidad que imponía la sociedad española. Mediante una compleja negociación entre el Ministerio de Información y Turismo, encargado del control de los medios de comunicación, y el Ministerio de Educación y Ciencia, se decidió regular los estudios de periodismo mediante una titulación genérica de Ciencias de la Información y una mención (o rama de especificidad) de periodismo. Al mismo tiempo, se abría la posibilidad de nuevas titulaciones mediante la aprobación del Decreto 2070/71 del 13 de agosto de 1971. Un mes más tarde se aprobaría el Decreto 2478/71 del 17 de septiembre que permitía la apertura de las dos primeras Facultades de Ciencias de la Información en la Universidad Autónoma de Barcelona, recién creada en 1968, y en la Universidad Complutense de Madrid. En 1972 se aprobarán, por parte del Ministerio de Educación y Ciencia, dos nuevas menciones de la titulación genérica: Publicidad y Relaciones Públicas y, de forma posterior, Imagen. Solo a partir de la reforma de los planes de estudio posteriores a 1984, y como consecuencia del nuevo marco universitario creado por la Ley de Reforma Universitaria (LRU) de 1982, se procedió a iniciar un proceso de constitución de tres titulaciones autónomas que culminarían a principios los años noventa: Periodismo, Comunicación Audiovisual y Publicidad y Relaciones Públicas.

La creación de las facultades de Ciencias de la Información implicará que estos centros universitarios constituyan el marco institucional específico en los que mayoritariamente se debata el ámbito disciplinar y se desarrolle la investigación, una vez iniciada la restauración democrática. En los años de la Transición democráticaª estas facultades centrarán su atención en la formación de los nuevos profesionales de la comunicación, consolidarán rápidamente su estatus universitario y, sobre todo, su reconocimiento social al servir y estar estrechamente unidas a dos de los actores esenciales en el proceso de construcción de la democracia: el periodismo y los nuevos medios de comunicación. Pero también, estas facultades, recién creadas y dotadas de medios materiales e institucionales muy escasos, iniciarán la definición del campo disciplinar mediante un arduo y complejo debate entre aquellos académicos que consideraban la formación profesional como el objetivo de los nuevos centros universitarios y aquellos otros, mayoritariamente surgidos de las primeras promociones de licenciados, que consideraban imprescindible la formación de la disciplina y el inicio de la investigación sobre medios y sistema comunicativo. Este debate, que resultó decisivo en los años posteriores entre "profesionalistas" y "comunicólogos", ha sido

1 La transición democrática es el periodo transcurrido entre la muerte del dictador Francisco Franco en 1975 y la aprobación de la Constitución democrática de 1978. En estos tres años de restauración de la monarquía, y bajo la presidencia de gobierno de Adolfo Suárez, se procedió al progresivo desmantelamiento de las estructuras políticas y sindicales del régimen dictatorial; la restauración en 1977 de la Generalitat de Catalunya, arraigada en la legitimidad republicana; la reforma democrática del sistema de medios y la generalización de las libertades democráticas que culminaron con la aprobación de la Constitución. La Transición democrática concluye con la aprobación de los estatutos de autonomía de Catalunya y Euskadi en 1979. 


\section{DISERTACIONES}

ENSAYOS

Anuario electrónico de estudios en Comunicación Social

ISSN: $1856-9536$

Doi: dx.doi.org/10.12804/disertaciones.09.02.2016.02

Volumen 9, Número 2 / julio-diciembre 2016

Versión PDF para imprimir desde

http://revistas.urosario.edu.co/index.php/disertaciones

descrito de forma pormenorizada por Martínez-Nicolás (2006) en uno de los textos de lectura obligada para conocer la formación de la investigación sobre periodismo, que constituyó la matriz del debate. El debate institucional sobre los objetivos de las facultades y los criterios de orientación de la investigación comunicativa se resolverán de forma definitiva en los años inmediatamente posteriores.

\section{Segundo marco institucional (1982-2002) (1): profesionalización y primera consolidación de la investigación comunicativa en España}

Este segundo periodo de institucionalización de la investigación se inició en 1982 con la aprobación de la Ley de Reforma Universitaria y culminará con la aprobación de la Agencia Nacional de Evaluación de la Calidad y Acreditación (ANECA) en 2002 y la posterior creación de la Comisión Nacional Evaluadora de la Actividad Investigadora (CNEAI).

La aprobación de la LRU fue decisiva para la investigación comunicativa española. Ofrecía nuevas posibilidades para el fomento de la actividad investigadora y la situaba como un elemento crucial para la proyección académica y el prestigio profesional de los universitarios. Josep Gifreu ha sido el investigador que de forma más explícita ha destacado el impacto de la LRU en este ámbito de investigación. En su informe sobre la investigación comunicativa en Cataluña, editado como monografía de la revista Anàlisi para su presentación en el Congreso de la AIERI en Barcelona (julio de 1988), describe de forma precisa cuatro innovaciones esenciales introducidas por la nueva ley (Gifreu, 1988). En primer lugar, la LRU creó los primeros sistemas de fomento de infraestructuras de investigación y el sistema de concurso para la asignación de proyectos competitivos. En este sentido, y se coincide con lo propuesto por Gifreu (1988), se produce una innovación crucial: "El proceso de consolidación de la Facultad de Ciencias de la Información la Universidad Autónoma de Barcelona, así como la aprobación en 1982 de la Ley de Reforma Universitaria (LRU), trajo una profesionalización progresiva de las primeras investigaciones universitarias en temas de comunicación" (p. 49). Una primera manifestación de esta tendencia hacia una cierta profesionalización en el ámbito de la investigación académica será un notable incremento de las tesis de doctorado presentadas en las facultades alcanzado una cifra espectacular de 93 tesis en el periodo comprendido entre 1993 y 1998 (Gifreu, 1988; Martínez-Nicolás, 2006).

Pero este periodo de los años ochenta y noventa no será decisivo solamente por los inicios de la profesionalización en la investigación comunicativa, sino que darán lugar a la formación del campo disciplinar mediante la introducción de los marcos teóricos que han configurado la investigación comunicativa internacional. Para el caso, fue la sociología el marco fuente a partir del cual se formó el ámbito. En palabras de Roberto de Miguel (2007), y en referencia a aquellos años,

Durante este periodo, los trabajos de Sociología de la comunicación más citados son los de Miquel de Moragas, Enric Saperas, Miguel Roiz, Manuel Martín Serrano, Blanca Muñoz, Julia Varela, Octavio Uña, Gonzalo Abril, Ángel Benito, Fermín Bouza, José Vidal Beneyto y Vicente Romano, entre otros (p. 588).

Este impulso de debate y formación del campo disciplinar fue decisivo para la configuración de la investigación comunicativa (Martínez-Nicolás, 2009) y, sobre todo, en la delimitación de los ámbitos de investigación más característicos (De-Miguel, 2007) ya en los años noventa. 


\section{DISERTACIONES}

ENSAYOS

Anuario electrónico de estudios en Comunicación Social

ISSN: $1856-9536$

Doi: dx.doi.org/10.12804/disertaciones.09.02.2016.02

Volumen 9, Número 2 / julio-diciembre 2016

Versión PDF para imprimir desde

http://revistas.urosario.edu.co/index.php/disertaciones

Por otra parte, estos veinte años implicarán un proceso de promoción de nuevos investigadores surgidos de las primeras promociones de las propias facultades que serán decisivos en la formación de los primeros grupos de investigación, encabezados por los investigadores seniors y fundacionales de la disciplina. La incorporación de las nuevas generaciones formadas en facultades del ámbito exclusivo de comunicación (Martínez-Nicolás, 2006) será el elemento que amalgame la investigación comunicativa y su primer periodo de consolidación, al mismo tiempo que dará lugar a creación de los primeros centros documentales del ámbito (Jones, 1997).

Por último, y este será un factor esencial que destaca Gifreu (1988) de forma muy precisa, la investigación comunicativa y la formación del campo disciplinar de la década de los ochenta no se hubiera producido sin la participación activa de la industria editorial del libro universitario, que en aquellos años procedió con una clara visión de futuro a la creación de las primeras colecciones especializadas en ciencias de la comunicación en sellos como Gustavo Gili, Ariel, Paidós o Mitre, entre otras.

La investigación comunicativa se había consolidado definitivamente después de veinte años de existencia de las facultades de comunicación (García-Jiménez, 2007; Jones, 1997, 1998; Martínez-Nicolás, 2006; Moragas, 1981; Rodrigo \& García-Jiménez, 2010). Y por consolidación se entiende tanto el reconocimiento y la promoción académica del investigador, como la creación de ciertos circuitos característicos de los profesionales de la investigación. La convergencia entre ambos factores institucionales se produjo en la década de los años ochenta. De forma más precisa, por consolidación se entiende que los profesionales de la investigación participan en la formación de investigadores en segundo y tercer ciclo universitario, se agrupan en asociaciones académicas internacionales y nacionales, su actividad se realiza mayoritariamente en grupos de investigación permanentes, compiten en convocatorias de proyectos de investigación o de infraestructura, gestionan contratos con empresas o fundaciones, producen conocimiento de forma regular que dan a conocer mediante la publicación de papers en revistas especializadas y en congresos organizados por asociaciones científicas especializadas en el campo disciplinar de la comunicación mediática. De forma intermitente, pero decidida, este circuito profesional, propio de la comunidad científica, se consolidó en España en aquella década.

\section{Segundo marco institucional (1982-2002) (2): Las licenciaturas de comunicación como titulaciones de éxito y su impacto sobre la consolidación (y algunas disfunciones) de la investigación comunicativa}

Como se ha visto, la investigación española en su conjunto recibirá un marcado impulso con la aprobación de la Ley Orgánica de Reforma Universitaria (LRU) que entrará en vigor en septiembre de 1983. Y la investigación comunicativa no será una excepción a este impulso. En su primer apartado, la Ley Orgánica definía el servicio público de la enseñanza superior y de sus académicos como la suma de tres funciones: el estudio, la enseñanza y la investigación. Por todo ello, cualquier profesional universitario debería ejercer necesariamente la docencia y la investigación. La aplicación de la nueva ley en las facultades de Ciencias de la Información fue decisiva por cuanto implicó tres tipos de reforma. En primer lugar, a partir 1984 se inició un decisivo proceso de reforma de los planes de estudio liderado mayoritariamente por las primeras promociones de profesorado formado en las facultades que constituían una segunda generación de profesores e investigadores. En esta reforma, como se ha comentado anteriormente, se 


\section{DISERTACIONES}

Anuario electrónico de estudios en Comunicación Social

ISSN: 1856-9536

Doi: dx.doi.org/10.12804/disertaciones.09.02.2016.02

Volumen 9, Número 2 / julio-diciembre 2016

Versión PDF para imprimir desde

http://revistas.urosario.edu.co/index.php/disertaciones

crearon tres titulaciones diferenciadas y mejor adaptadas a las necesidades de un mercado profesional en democracia: Periodismo, Comunicación Audiovisual y Publicidad y Relaciones Públicas. En segundo lugar, la investigación comunicativa por vez primera era considerada como una prioridad en sus centros universitarios. Y, por último, la reforma de los planes de estudio mayoritariamente introducían una clara novedad al promover las ciencias sociales como ámbito de referencia de la investigación comunicativa que se constituía como un campo disciplinar específico en el conjunto de las teorías sociales. Consecuencia de ello fue el cambio en la denominación de los centros universitarios, ahora facultades de Ciencias de la Comunicación. Esta nueva denominación reclamaba no solo una formación profesional que abarcaba todas las profesiones comunicativas sino, y de forma más significativa, un campo de investigación propio a desarrollar en las nuevas facultades desde una perspectiva pluridisciplinar.

El éxito de la reforma fue inmediato. Profesionalmente su éxito fue innegable por cuanto consiguieron ser aceptadas como un factor de referencia en la formación de los profesionales de la comunicación en España, siendo su presencia extraordinariamente mayoritaria en los profesionales del periodismo, de la producción y realización audiovisuales, en los profesionales de la publicidad y, de forma indiscutible, en las relaciones públicas y la comunicación empresarial. En cuanto a la investigación, la reforma fue decisiva para su desarrollo inmediato por cuanto vinculó su estructura y organización a las nuevas titulaciones. Estas serán decisivas para discutir el campo disciplinar, definir los límites de cada especialidad, ordenar los objetos de estudio y establecer áreas de conocimiento.

A pesar de todas las reticencias de ciertos sectores universitarios y las demostraciones de desatención por una parte significativa de sociólogos, psicólogos, pedagogos o lingüistas, las titulaciones de comunicación consiguieron un reconocimiento social y constituyen una alternativa atractiva para miles de estudiantes en cada nuevo curso académico. Los datos son abrumadores. En 1982 contábamos con cuatro facultades, un título genérico y tres menciones. Estos cuatro centros se situaban en la Universidad Autónoma de Barcelona, la Universidad Complutense de Madrid, la Universidad de Navarra (universidad privada del Opus Dei) y la Universidad del País Vasco - Euskal Herriko Unibertsitatea, y el número de profesores adscritos era aproximadamente de 500 (Jones, 1998; Martínez-Nicolás, 2006). En 1998 el número de facultades era de 30, de las cuales 10 eran privadas, se habían creado las tres titulaciones diferenciadas, el número de alumnos era aproximadamente de 20000 y el número de profesores se acercaba a los 2000 (Martínez-Nicolás, 2006). En el cuarenta aniversario de los primeros centros, en 2015, en España existían 54 facultades con titulaciones específicas de comunicación, de las cuales 35 son públicas y 19, privadas, que agrupan un total aproximado de 45000 alumnos. El número de profesores es incierto, pero los datos no oficiales sitúan su número total en la actualidad cerca de los 4200 docentes e investigadores.

Esta situación de éxito ha sido determinante para situar estas facultades entre las de mayor demanda en ciencias sociales en el sistema universitario español. Sin embargo, una situación compleja como la que se acaba de describir y con un crecimiento tan acelerado de centros y de personal de docencia y de investigación ha conllevado ciertas disfunciones. Como comentaba Daniel Jones en 1997, se había producido un excesivo número de alumnos, una inevitable deficiencia en la formación de un sector del profesorado, a menudo una insuficiente dotación de equipos técnicos y una cierta inadecuación de los planes de estudio respecto a las demandas sociales y laborales (Jones, 1997). En todo caso, un dato que se debe tener muy en cuenta es que el número de académicos encuadrados en las facultades de comunicación excede de forma muy notable a la del resto de centros universitarios en ciencias sociales y humanidades y suele corresponderse con nuestro entorno internacional. Daniel Jones (1998) diagnosticaba con ironía esta situación con las siguientes palabras: 


\section{DISERTACIONES}

Ensayos

Anuario electrónico de estudios en Comunicación Social

ISSN: $1856-9536$

Doi: dx.doi.org/10.12804/disertaciones.09.02.2016.02

Volumen 9, Número 2 / julio-diciembre 2016

Versión PDF para imprimir desde

http://revistas.urosario.edu.co/index.php/disertaciones

España ha logrado un nivel muy alto en el panorama internacional por lo que respecta al número de profesionales en el estudio de los propios medios de comunicación y de las industrias culturales ('comunicólogos'), unos por vocación y otros abocados a este campo académico por la presión laboral. Al aumentar el número de facultades y de estudiantes fue necesaria la contratación exponencial de nuevo personal docente - preparado o no-, hecho que ha llevado a un crecimiento exponencial del número de personas que se dedican profesionalmente -en exclusividad o a tiempo parcial- a este ámbito de estudios (p. 22).

\section{Tercer marco institucional (2002-2015): la creación del espacio europeo de enseñanza superior y la introducción del estándar internacional}

La LRU tenía como finalidad forzar la modernización de la universidad española mediante una única estrategia a medio y largo plazo: adaptar los centros universitarios españoles a los estándares internacionales y la promoción de su presencia internacional. Este decidido esfuerzo por dejar atrás un sistema obsoleto y con escasa influencia externa coincidió con la formación en el ámbito internacional de un nuevo tipo de estándar universitario internacional. Ambas realidades - la voluntad de modernización y la creación de un nuevo estándar - han determinado un nuevo marco institucional al que actualmente la investigación comunicativa intenta adaptarse de forma esforzada. Esta tercera institucionalización, si se hace referencia al ámbito internacional en el que ha surgido, se ha estructurado mediante cuatro actores que dominan y definen el campo disciplinar: agencias de calificación universitarias, revistas nacionales e internacionales del ámbito, asociaciones académicas y la industria editorial británica y norteamericana. Nuevos actores como las agencias de calificación (por ejemplo) y actores tradicionales pero ahora con un nuevo carácter de centralidad como las revistas y las asociaciones (por ejemplo) pasarán a centrar el debate metodológico y la definición del campo disciplinar.

En el caso español, sin duda la novedad más determinante en la neoinstitucionalización de la investigación fue la creación de las agencias de calificación destinadas preferentemente a la evaluación de la calidad científica y académica y a los sistemas de cálculo de la producción científica a partir de la producción, gestión y objetivación de resultados a partir de criterios establecidos por la administración pública. En España se ha producido su introducción de forma más intensa y acelerada que en Portugal e Italia, y de forma mucho más decidida que en Francia. La puesta en marcha en 2002 de la Agencia Nacional de Evaluación de la Calidad y Acreditación (ANECA), competente en titulaciones universitarias, acreditaciones, verificaciones de títulos, y la posterior creación de la Comisión Nacional Evaluadora de la Actividad Investigadora (CNEAI), competente en la evaluación de la producción científica y en calidad de la investigación, y de las agencias de calidad autonómicas ${ }^{2}$ configuran el núcleo del nuevo marco

2 Cataluña: Agència per a la Qualitat del Sistema Universitari (AQU); Islas Baleares: Agència de Qualitat Universitària (AQUIB); Canarias: Agencia Canaria de Evaluación de la Calidad y Acreditación Universitaria (ACECAU); Andalucía: Agencia Andaluza de Evaluación de la Calidad y Acreditación, Unidad para la Calidad de las Universidades Andaluzas (UCuA); Castilla y León: Agencia para la Calidad del Sistema Educativo Universitario; Galicia: Axencia para a Calidade do Sistema Universitario de Galicia (AcsuG); Comunidad Valenciana: Comisión Valenciana de la Acreditación y Evaluación de la Calidad; País Vasco: Agencia de Evaluación y Acreditación de la Calidad del Sistema Universitario; y Aragón: Agencia de Calidad y Prospectiva Universitaria de Aragón. 


\section{DISERTACIONES}

ENSAYOS

Anuario electrónico de estudios en Comunicación Social

ISSN: 1856-9536

Doi: dx.doi.org/10.12804/disertaciones.09.02.2016.02

Volumen 9, Número 2 / julio-diciembre 2016

Versión PDF para imprimir desde

institucional totalmente novedoso en la universidad española y acorde con los criterios de la reforma del espacio europeo de enseñanza superior (EEES), cuya implantación definitiva se producirá entre 2008 y 2012. Todo este proceso ha implicado un cambio de primer orden: la investigación en comunicación institucionalmente pasará a integrase en el conjunto de las ciencias sociales en el que compite como un área plenamente consolidada y externa a las facultades de Ciencias de la Comunicación.

Para la investigación comunicativa, las consecuencias de la implantación de este modelo de orden europeo, pero adaptado a las tradicionales estructuras administrativas españolas, han sido múltiples y de una extraordinaria diversificación. La introducción de agencias de calificación era una acción necesaria que permitía establecer la internacionalización de la investigación y el incremento de su competitividad como objetivos básicos irrenunciables. Pero su presencia probablemente no ha sido neutral y ha priorizado ciertas modalidades de investigación más susceptibles de adaptarse a ciertos criterios de cálculo y de gestión administrativa. Para el ámbito, el debate y la consideración de los pros y contras de este tipo de modelo se inició ya en el I Congreso de la Asociación Española de Investigación de la Comunicación (AE-IC) que tuvo lugar en Santiago de Compostela. Entre algunas comunicaciones sobre este nuevo sistema destacaba la propuesta de un 'Efecto ANECA' que proponía Jaume Soriano de la Universidad Autónoma de Barcelona y que definía con estas palabras:

Desde la aparición de las primeras agencias autonómicas hasta el recién publicado decreto que delega en

la ANECA la capacidad para acreditar a los candidatos a los cuerpos docentes universitarios del estado, la

joven comunidad del campo de la investigación en comunicación ha ido virando hacia un tipo de produc-

ción científica que satisfaga más a las exigencias de reconocimiento de dichas agencias que a la inercia de

la tradición investigadora heredada hasta ahora (Soriano, 2008, p. 1).

Sin entrar en debatir la propuesta de Jaume Soriano, acertada en sus criterios generales, el 'efecto' propuesto deber ser considerado como un elemento determinante para comprender la investigación comunicativa española por la mejora de los procesos de evaluación de la calidad de la investigación comunicativa en España (Silva \& San-Eugenio, 2014), por un impulso del incremento de la presencia española en revistas y sistemas de citas internacionales (Masip, 2011) o por la creación de estructuras más eficientes en la práctica profesional de la investigación (Piñuel, Lozano \& García-Jiménez, 2011). Pero también, sin duda los criterios administrativos de estas agencias han influido en la investigación reciente al menos en dos aspectos: la adopción del empirismo cuantitativo y estadístico como patrón central de la investigación y la pérdida de 'valor académico' de la investigación crítica y de la investigación de carácter comprensivo. En definitiva, probablemente y como consecuencia de la implantación de este nuevo modelo los investigadores españoles son mucho más capaces de explicar todo tipo de segmentos empíricos que en décadas anteriores, pero con menor capacidad para comprender e intervenir en el sistema de medios.

Pero esta nueva institucionalización exige el desarrollo robusto de otros dos actores imprescindibles: las revistas especializadas en comunicación y las asociaciones académicas y de profesionales de la investigación. Ambas instituciones son centrales y, aunque su desarrollo ha sido notable, en España actualmente nos encontramos en un periodo marcado por la necesidad de consolidar su protagonismo y su influencia en la investigación española, y lo que es más significativo, se enfrentan a un reto decisivo como es asumir como propios y alcanzar los estándares internacionales. 


\section{DISERTACIONES}

ENSAYOS

Anuario electrónico de estudios en Comunicación Social

ISSN: 1856-9536

Doi: dx.doi.org/10.12804/disertaciones.09.02.2016.02

Volumen 9, Número 2 / julio-diciembre 2016

Versión PDF para imprimir desde

Las revistas especializadas han conseguido ser una institución decisiva en la profesionalización de la investigación. No se puede acceder al estatus de profesional de la investigación ni conseguir las evaluaciones de las agencias de cualificación sin una presencia regular en las revistas especializadas. En España las revistas en comunicación son relativamente pocas si se las compara con las de otras ciencias sociales aunque su crecimiento ha sido muy notable pasando de 23 en 2010 a 69 en 2015. Del conjunto de estas revistas, actualmente solo 24 cuentan con un funcionamiento ordinario y unos plazos de edición regulares. Las revistas fuente todavía constituyen una agrupación muy restringida y este constituye uno de los problemas más agudos de la investigación comunicativa. Por revistas fuente se entiende "un pequeño grupo que origina o produce el $80 \%$ de las citas de la especialidad y son las utilizadas para hallar el factor de impacto, sin necesidad de acudir a una muestra universal" (De-Pablos, 2011, p. 12). Las revistas fuente forman un grupo de 6 publicaciones (Comunicación \& Sociedad, Estudios del Mensaje periodístico, Revista Latina de Comunicación Social, Comunicar, zer y Anàlisi), del total de 69, que se podría ampliar a 3 publicaciones en proceso de integrarse en este reducido grupo. En todo caso, es necesario diversificar este número de revistas debido a que, con una comunidad académica superior a los 4000 docentes e investigadores, resulta imposible atender a toda la demanda de publicación de papers. Si a esto se añade que las agencias de calificación están incrementando los criterios solicitados asimilándolos a los sistemas de calificación internacionales, la disfunción se produce de forma inevitable y en crecimiento. Esta situación disfuncional se agrava si se tiene en cuenta que en la mayoría de ciencias sociales españolas la presencia de revistas fuentes es superior con comunidades académicas menores (De-Pablos, 2011).

En este contexto de mayor relevancia institucional de las revistas del campo no es de extrañar que la investigación sobre estas publicaciones haya generado en los últimos años una significativa producción de estudios sobre las revistas científicas y análisis bibliométricos que evalúan la situación de la investigación española, atendiendo a aspectos como la situación general de la investigación en comunicación en España (Martínez-Nicolás, 2008; Piñuel, Lozano \& García, 2011), la propia producción científica por medio sus artículos (Cortiñas-Rovira \& Escribà-Sales, 2013; Fernández-Quijada, 2011; López-Rabadán \& Vicente-Mariño, 2011; Martínez-Nicolás \& Saperas, 2011), los criterios de evaluación y posicionamiento de sus publicaciones (Castillo, Rubio \& Almansa, 2012), la presencia internacional de autores y editores españoles (De Filippo, 2013; Fernández-Quijada, Masip \& Berguillos, 2013), e incluso el papel de las publicaciones científicas en la difusión del conocimiento (De Pablos, 2010; Castillo, 2011).

El último elemento institucional de la investigación lo constituyen las asociaciones académicas. Las asociaciones en España aparecen en la década de los años noventa como consecuencia de la formación de los primeros grupos de investigación y de su consolidación y en el mismo periodo que la mayor parte de las revistas fuente. En 1990 se creó la Sociedad Española de Periodística y dos años más tarde se creó la Asociación de Historiadores de la Comunicación. A estas dos primeras asociaciones se les añadió en 2005 la Asociación de Investigadores en Relaciones Públicas (AIRP), en 2008 se creó la Asociación de Comunicación Política (ACOP) y en 2010 la Sociedad Latina de Comunicación Social. Si todas estas asociaciones son de carácter sectorial, en 2006 la conferencia de decanos de las facultades de Ciencias de la Comunicación impulsó la asamblea fundacional de la primera asociación genérica, la Asociación Española de Investigadores de la Comunicación (AE-IC) que en enero de 2008 realizaría su primer

3 Para la consulta de las revistas españolas de comunicación véase El Observatorio para las Revistas Científicas en http://www.observatorioderevistascientificas.com/index.php/revistas/comunicacion 


\section{DISERTACIONES}

ENSAYOS

Anuario electrónico de estudios en Comunicación Social

ISSN: 1856-9536

Doi: dx.doi.org/10.12804/disertaciones.09.02.2016.02

Volumen 9, Número 2 / julio-diciembre 2016

Versión PDF para imprimir desde

http://revistas.urosario.edu.co/index.php/disertaciones

congreso en Santiago de Compostela. A estas asociaciones deben sumarse dos instituciones con carácter de academia científica. En 1984 se creó en Cataluña la Societat Catalana de Comunicació de l'Institut d'Estudis Catalans (SCC-IEC) y en el año 2000 se constituía la Sociedad Galega de Investigadores e Investigadoras en Comunicación (AGACOM) (Moragas, 2014).

La actualización del papel y la funcionalidad de las asociaciones constituyen otro reto central de la investigación comunicativa que deberá resolverse en los próximos años. Se trata de una asignatura pendiente no tanto por una carencia de asociaciones representativas de la investigación española sino por la necesidad de superar el objetivo exclusivo de la realización de congresos y de facilitar el intercambio científico entre expertos y grupos de investigación. Estas asociaciones internacionales y nacionales, sectoriales o genéricas han pasado a ser el marco institucional que de forma más intensa ha modificado la organización intelectual de la disciplina. Cumplen, entre otros objetivos, una función de organización académica, de taxonomía de los objetos de estudio (secciones temáticas), de debate y pugna por el dominio en el campo disciplinar, de reputación profesional y de relevancia de los centros universitarios y de los grandes grupos de investigación. Y naturalmente, en algunos casos, son la matriz de algunas de las grandes publicaciones. Esta necesidad de incrementar la presencia de las asociaciones en el campo disciplinar y en las políticas científicas y de comunicación y coordinar su acción con las grandes asociaciones internacionales fue puesta en debate conjuntamente con representantes de asociaciones internacionales, al parecer, por vez primera en España, como actividad pre-congresual, en el iv Congreso de la AE-Ic que tuvo lugar en enero de 2014 en Bilbao. En los tres días de comunicaciones y de debate ${ }^{4}$ se propusieron soluciones efectivas para resituar las asociaciones en el complejo papel institucional que les corresponde. De forma especialmente significativa en el texto titulado "Las asociaciones de investigación de la comunicación. Funciones y retos" de Miquel de Moragas (2014) se proponen diez funciones que deben asumir estas asociaciones de forma similar a las ejercidas por las grandes asociaciones internacionales (Moragas, 2014). Y este es un desafío que, al igual que el planteado por el número de las revistas fuentes, debe ser resuelto de forma efectiva en los próximos años.

\section{Un reto de futuro: la internacionalización}

Se finaliza este ensayo, que ha tenido como punto de mira los procesos de institucionalización y de profesionalización en la investigación comunicativa española. Naturalmente, un análisis completo del campo disciplinar solo hubiera sido posible mediante la consideración de un segundo aspecto que no se trató en estas páginas: la construcción del campo disciplinar, los marcos teóricos y los métodos aplicados por los investigadores, sus competencias técnicas, su producción científica, los objetos de estudio, la formación y funcionamiento de los grupos de investigación, las modalidades de autoría y la colaboración con investigadores internacionales.

En este caso solo se ha observado el contexto institucional. Del conjunto de este análisis se concluye que la investigación española sobre comunicación mediática se define por ser un sector de las ciencias sociales plenamente

4 Las comunicaciones de M. de Moragas, M. Vicente-Mariño, K. Nordenstreng, A. García Jiménez, M. Felici y M.L. Humanes en el "Encuentro internacional de asociaciones académicas de comunicación. Razón de ser y retos en la globalización” (AE-IC. 19,20 y 21 de enero, Bilbao) pueden consultarse en http://www.ae-ic.org/ esp/actualitat_det.asp?id_seccio=4\&id_noticies=192

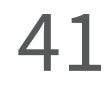




\section{DISERTACIONES}

ENSAYOS

Anuario electrónico de estudios en Comunicación Social

ISSN: 1856-9536

Doi: dx.doi.org/10.12804/disertaciones.09.02.2016.02

Volumen 9, Número 2 / julio-diciembre 2016

Versión PDF para imprimir desde

consolidado y asociado con centros universitarios que cuentan con titulaciones de éxito social y profesional. Y este es el principal rasgo diferencial respecto a los países del entorno más cercano. El protagonismo de las facultades de Ciencias de la Información y de la Comunicación tanto en la formación del campo disciplinar como en la formación profesional de los alumnos. Aunque la intervención de otras ciencias sociales ha sido una realidad constante en la investigación española, lo ha sido de forma minoritaria tanto en volumen de investigación como en facultades ajenas a la comunicación mediática. Este es un segundo rasgo diferencial de la investigación española al no contar con una presencia relevante de las facultades y departamentos de ciencias sociales y de humanidades. Esta ausencia también ha influido en la formación de los investigadores, al menos hasta la última generación incorporada en el ámbito. El debate sobre el papel que deben cumplir las ciencias sociales en la formación de investigadores para evitar una tendencia hacia una "autarquía" metodológica y teórica es un debate que todavía está pendiente, pero se trata de un debate crucial que afecta a la definición de competencias de nuestros investigadores a menudo más interesados en los objetos de estudio que en los marcos teóricos a partir de los cuales se puede acceder a su observación.

Se trata, pues, de una investigación consolidada, aunque no madura. Aunque como ya se ha afirmado anteriormente, todavía queda por reforzar la función que cumplen las revistas especializadas y las asociaciones académicas. En el primer caso, se está ante una dificultad de difícil solución al disponer de pocas revistas fuente para una comunidad de investigadores formada por miles de efectivos y en un momento en que las mismas revistas fuente se ponen en duda como promoción profesional por las agencias de calificación que sitúan en la indexación internacional el criterio fundamental de evaluación. En el segundo caso, las asociaciones de profesionales de la investigación se encuentran ante el reto de asumir capacidades de organización de campo disciplinary de referencia en las políticas de investigación siguiendo la estela de las asociaciones internacionales.

Se trata, pues de una investigación consolidada que debe dar pasos decididos para su maduración definitiva. Quizá se puedan discutir cuáles son sus mayores déficits y qué acciones hay que emprender. Pero probablemente se sabe cuál es el destino que hay que alcanzar en los próximos años: la internacionalización. En 1997 la revista Anàlisi publicó un excelente número editado por Manuel Parés dedicado a la "investigación europea en comunicación social". En cada artículo encargado a una personalidad relevante se plantaban cinco "líneas de actuación" a manera de patrón general que debía responder cada autor. La última propuesta era la siguiente: "[describir las] influencias externas, así como la influencia ejercida más allá de las fronteras" (Parés, 1997, p. 25). La investigación solo podrá madurar y crear masa crítica efectiva cuando sea capaz de poder responder de forma positiva a la presencia de ambas formas de influencia: del exterior hacia nosotros y de nosotros hacia el exterior. La internacionalización es el reto fundamental cuya respuesta es ineludible.

\section{Referencias}

1. Almirón, N., \& Reig, R. (2007). The Communications Research in Spain: the Political Economy. Epsitemological Approach. American Communication Journal, 9(2), 51-67.

2. Berrio, J. (1997). Un segle de recerca sobre comunicación a Catalunya. Estudi crític dels principals àmbits d'investigació de la comunicació de massa. Bellaterra, Cerdanyola: Edicions Universidad Autónoma de Barcelona. 


\section{DISERTACIONES}

ENSAYOS

Anuario electrónico de estudios en Comunicación Social

ISSN: 1856-9536

Doi: dx.doi.org/10.12804/disertaciones.09.02.2016.02

Volumen 9, Número 2 / julio-diciembre 2016

Versión PDF para imprimir desde

3. Calhoum, C. (2011). Communication as Social Science (and More). International Journal of Communication, 4, 1479-1496.

4. Castillo A. (2011). El rol de las publicaciones científicas en Comunicación en el eeEs: indexación e impacto. Revista Internacional de Relaciones Públicas, 1(1), 135-154.

5. Castillo, A., Rubio, A. L., \& Almansa, A. (2012). La investigación en Comunicación. Análisis bibliométrico de las revistas de mayor impacto del ISI. Revista Latina de Comunicación Social, 67, 248-270.

6. Cortiñas-Rovira, S., \& Escribà-Sales, E. (2013). La internacionalización y las coautorías en las principales revistas científicas de Comunicación en España. Comunicar, 21(41).

7. De-Filippo, D. (2013). La producción científica española en Comunicación en wos. Las revistas indexadas en ssci (2007-12). Comunicar, 21(41), 25-34.

8. De-Miguel, R. (2007). Sociología de la comunicación. En M. Pérez Iruela, (Comp). La sociología en España (pp. 583-607). Madrid: Centro de Investigaciones Sociológicas (CIS) y Federación Española de Sociología (FES).

9. De-Pablos, J. M. (2010). Análisis de las revistas españolas de comunicación, tras la actualización del índice de impacto de 2009.En // Congreso Internacional Latino de Comunicación Social. La Laguna (Tenerife): Sociedad Latina de Comunicación Social.

10. De-Pablos, J.M. (2011). Las revistas científicas de comunicación. Documento de Trabajo. En J. L. Piñuel; C. Lozano y A. García (eds.): Investigar la comunicación en España. Madrid: AE-IC.

11. Fernández-Quijada, D. (2011). De los investigadores a las redes: una aproximación tipológica a la autoría en las revistas españolas de comunicación. En J. L. Piñuel, C. Lozano \& A. García (Eds.), Investigar la comunicación en España (pp. 633-648). Madrid: AE-IC.

12. Fernández-Quijada, D., \& Masip, P. (2013). Tres décadas de investigaciòn espanyola en comunicación: hacia la mayoría de edad. Comunica, 41, 15-24.

13. Fernández-Quijada, D., Masip, P., \& Bergillos, I. (2013). El precio de la internacionalidad: la dualidad en los patrones de publicación de los investigadores españoles en comunicación. Revista Española de Documentación Científica, 36(2), 111-123.

14. García-Jiménez, L. (2007). Las teorías de la comunicación en España: un mapa sobre el territorio de nuestra investigación (1980-2006). Madrid: Tecnos

15. Gifreu, J. (1988). Mass Communication Research in Catalonia. Anàlisi, Monográfico. 1-137.

16. Humanes, M. L. (2007). La investigación sobre la profesión periodística en España. Sphera publica, Número Extra 1, 173-194.

17. Jones, D. (1997). Investigació sobre comunicación social a l'Espanya de les autonomies. Anàlisi, 21, 101-120.

18. Jones, D. (1998). Investigación sobre comunicación en España: Evolución y perspectivas. ZER, 5, 13-51.

19. López-Rabadán, P., \& Vicente-Mariño, M. (2011). Métodos y técnicas de investigación dominantes en las Revistas científicas españolas sobre comunicación (2000-2009). En J. L. Piñuel, C. Lozano \& A. García (eds.), Investigar la comunicación en España. (pp. 665-679). Madrid: AE-Ic.

20. Martínez-Nicolás, M. (2006). Masa (en situación) crítica. La investigación sobre periodismo en España: comunidad científica e intereses de conocimiento. Anàlisi, 33, 135-170. 


\section{DISERTACIONES}

ENSAYOS

Anuario electrónico de estudios en Comunicación Social

ISSN: 1856-9536

Doi: dx.doi.org/10.12804/disertaciones.09.02.2016.02

Volumen 9, Número 2 / julio-diciembre 2016

Versión PDF para imprimir desde

http://revistas.urosario.edu.co/index.php/disertaciones

21. Martínez-Nicolás, M. (2008). La investigación sobre comunicación en España. Evolución histórica y retos actuales. En M. Martínez-Nicolás, (Coord.), Para investigar la comunicación. Propuestas teórico-metodológicas. Madrid: Tecnos, 13-52.

22. Martínez Nicolás, M., \& Saperas, E. (2011). La investigación sobre Comunicación en España (1998-2007). Análisis de los artículos publicados en revistas científicas. Revista Latina de Comunicación Social, 66, 101-129.

23. Masip, P. (2011). Los efectos del Efecto Aneca: Análisis de la Producción Española en comunicación en el Social Sciences Citation Index. Revista Comunicar. Recuperado de http://www.revistacomunicar.com/ pdf/2011-04-Masip.pdf

24. Moragas, M. (1981). Teorías de la comunicación. Investigaciones sobre medios en América y Europa. Barcelona: Gustavo Gili

25. Moragas, M. (2000). Estructura i polítiques de comunicación. En Departament de Periodismo i Ciències de la comunicación (UAB), Universidad i Periodisme. Actes de les jornades sobre continguts acadèmics $i$ docència a la llicenciatura de Periodisme (pp. 45-48). Bellaterra, Cerdanyola: Ediciones de la Universidad Autónoma de Barcelona.

26. Moragas, M. (2014). Las asociaciones de investigación de la comunicación. Funciones y retos. Ponencia presentada en iv Congreso de la Asociación Españolas de Investigadores en Comunicación (AE-IC). Encuentro precongresual "Encuentro internacional de asociaciones académicas de comunicación. Razón de ser y retos en la globalización”. Bilbao, 20 de enero. Recuperado en http://www.ae-ic.org/esp/actualitat_det. asp?id_seccio=4\&id_noticies $=192$

27. Parés, M. (1997). La recerca europea en comunicación social. Introducció. Anàlisi, 21.

28. Piñuel, J. L. (Ed.) (2011). La investigación y la docencia universitarias entorno a la Comunicación como objeto de estudio en Europa y en América Latina. La Laguna, Tenerife: Cuadernos artesanos de Latina, 15.

29. Piñuel, J. L., Lozano, C., \& García-Jiménez, A. (Eds.) (2011). Investigar la comunicación en España. Madrid: AE-Ic.

30. Rodrigo, M., \& García-Jiménez, L. (2010). Communication Theory and Research in Spain: A Paradigmatic Case of a socio-humanistic discipline. European Journal of Communication, 25(3), 273-286.

31. Salaverría, R. (2007). La investigación sobre ciberperiodismo en España: tedencias, resultados y perspectivas. En Jornada "La convergencia digital en los medios de comunicación valencianos". Departament de Teoria dels Llenguatges i Ciències de la Comunicació. Universidad de València. Valencia. Recuperado de: https://www.academia.edu/673095/La_investigacion_sobre_ciberperiodismo_en_Espana_tendencias_ resultados_y_perspectivas.

32. Saperas, E. (1997). Introducció a les teories de la comunicació. Barcelona: Editorial Pòrtic.

33. Saperas, E., \& Carrasco-Campos. (2015). The operationalization of the concept of framing in the Journal of Communication (2009-2013). Objects of study, research techniques and theoretical construction. Communication \& Society 28(4), 49-66.

34. Silva, V., \& San-Eugenio, J. (2014). La investigación en comunicación ante una encrucijada: de la teoría de los campos a la diseminación y diversidad gnoseológica. Estudio inicial comparado entre España, Brasil y Chile. Palabra Clave, 17(3), 803-827.

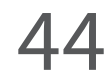




\section{DISERTACIONES}

ENSAYOS

Anuario electrónico de estudios en Comunicación Social

ISSN: 1856-9536

Doi: dx.doi.org/10.12804/disertaciones.09.02.2016.02

Volumen 9, Número 2 / julio-diciembre 2016

Versión PDF para imprimir desde

http://revistas.urosario.edu.co/index.php/disertaciones

35. Soriano, J. (2008). El efecto ANECA. Ponencia presentada en I Congreso Internacional de la Asociación de Investidores de la Comunicación (AE-IC). Santiago de Compostela. Recuperado de: http://www.ae-ic.org/ santiago2008/contents/pdf/comunicaciones/286.pdf 